International Journal on Cybernetics \& Informatics (IJCI) Vol. 3, No. 3, June 2014

\title{
Classifier Fusion Method to Recognize HANDWRitTen PERsian Numerals
}

\author{
Reza Azad $^{1}$, Babak Azad $^{2}$, Iraj Mogharreb ${ }^{3}$, Shahram Jamali $^{4}$ \\ ${ }^{1}$ Department of Electrical and Computer Engineering, Shahid Rajaee Teacher Training \\ University, Tehran, Iran \\ ${ }^{2}$ Computer Engineering Department, University of Mohaghegh Ardabili, Ardabil, Iran \\ ${ }^{3}$ Ardabil Branch Islamic Azad University, Ardebil, Iran \\ ${ }^{4}$ Associate professor, Faculty of Computer Engineering, University of Mohaghegh \\ Ardabili, Ardabil, Iran
}

\begin{abstract}
Recognition of Persian handwritten characters has been considered as a significant field of research for the last few years under pattern analysing technique. In this paper, a new approach for robust handwritten Persian numerals recognition using strong feature set and a classifier fusion method is scrutinized to increase the recognition percentage. For implementing the classifier fusion technique, we have considered $k$ nearest neighbour $(K N N)$, linear classifier $(L C)$ and support vector machine (SVM) classifiers. The innovation of this tactic is to attain better precision with few features using classifier fusion method. For evaluation of the proposed method we considered a Persian numerals database with 20,000 handwritten samples. Spending 15,000 samples for training stage, we verified our technique on other 5,000 samples, and the correct recognition ratio achievedapproximately 99.90\%. Additional, we got $99.97 \%$ exactness using four-fold cross validation procedure on 20,000 databases.
\end{abstract}

\section{KEYWORDS}

Persian handwritten recognition, $k$ nearest neighbor, linear classifier, SVM classifier, classifier fusion.

\section{INTRODUCTION}

Nowadays handwritten characters recognition is one of the most popular research areas, because it has various application potentials. Bank cheques processing, Postal Automation, Automatic data entry, etc. are some of its potential application are. Most of the handwritten character recognition methods for, Arabic, English, and Chinese scripts are reviewed in [1-3]. As regards there is no popular method for Persian handwritten character recognition due to cursive-ness of Persian handwritten, and various way of characters combination together and also, characters position in words. By the following passages, we studied edge maps, transit and directional frequencies effect in the numeral image contour pixels as features, which kept morphological information of input and then applied fusion of classifiers as classifier.

In Iran and some of its neighbouring countries, the Persian numerals have usage. Also, the Persian has 10 numerals. Alphabets of Persian and Arabic scripts are written from right to left but in their text, digits are taken place from left to right. Despite the similarity of Persian and Arabic numerals, there are a few important differences between their scripts [4]. Normally, in Persian

DOI: 10.5121/ijci.2014.3301 
there are two types of writing for the digits $0,2,4,5$ and 6 . These characteristics make Persian numerals recognition so sophisticate than other languages [4]. Fig. 1 shows the Examples of Persian printed and handwritten digits.

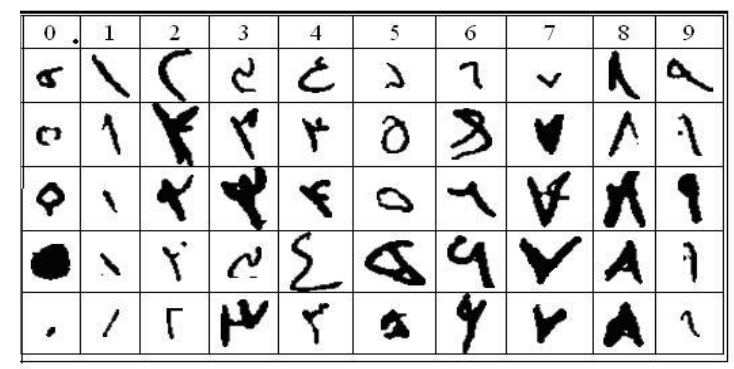

Figure 1. Sample of Persian Handwritten numerals [4]

In the related workspertinent to the Persian handwritten character recognition, many approaches for feature extraction and classificationhas been proposed. Some of the latest methods in field of feature extraction are; shadow and segmentation codes [5-7], fractal approach [8], profiles [9], moment features [10], template matching [11], structural feature set [12] and wavelet [13], [14]. Also for classification stage different types of Neural Networks [5-8], [10], [11], SVM's [9], [13], [15], Nearest Neighbour [12], multiple classifier [16], [17] have been implemented. What achieved from the literature survey on Persian handwritten character recognition, it is clear that not much attempt was increased to recognize a more capable feature set (some of them are time consuming and some of them cannot keep the structure of the input image for feature extraction stage), which could more suitably be respond to the recognition part [4]. For solving this kind of issue, we investigatedmore robust featureswith use of transit, edge maps and modified contour chain code of every window-map, and then apply fusion of classifiers for classification. This kind of feature set, which explicit the somatic shape of input character and take out local information of the input image in each window-map, provided very good correctness in experimental stage.

We ought toremarkthat the suggested system did not use any pre-processing methods (skew and tilt detection/improvement, smooth out, noise elimination, etc.) that were luxuriousprocesses. Likewise, strength of our feature set was under treatment some of these issues such as skew and slant reasonably.

The rest of our paper is organized as follows: feature extraction technique is detailed in Section two, classification stage id described in Section three, experimental results and comparative investigation are labelled in Section four and finally in last section we assign the conclusion.

\section{FEATURE EXTRACTION TEChNIQUE}

In this phase we will extract chain-code, modified edge maps and transition feature set. Extracting tactic of these feature set are detailed in the next subsections.

\subsection{Directional Chain Code FrequenciesFeature Set}

Directional chain code frequencies of the outline points of the input image are very useful for different application such as character segmentation, recognition, etc. [18]. In our proposed method as a first feature set we extracted chain-code directional frequencies of outline pixels of the images by the following rule: First the minimum rectangle covering the handwritten character (bounding box) is extracted for every input image, Then for removing the features to size and 
position, we adapted each image to a normal size of $49 \times 49$ pixels. We selected this normalized value based of manyexperimentations and a geometric study. Fig. 2(a) shows the normalized image with its covering rectangle and in Fig. 2(b) the extracted outline points of the character is shown.

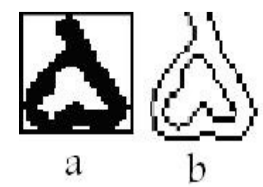

Figure 2. (a): bordering of a normalized image (b): Digit '5' outlineshape

By possession a window-map of size $7 \times 7$ on the image, we scanned the image outline horizontally from the top left maximum point to flatmaximum point (that contains 49 no overlapped blocks) and we extracted a8 directions chain code frequencies for each block (8 directions were depicted in Fig. 3(a)). As a replacementextracting the feature set in terms of 8 orders, we have offered to simplify the features into 4 sets fit to 4 orders (Fig. 3(b) shows the four directions), that the horizontal direction code are determined by the 0 and 4 directions, vertical direction code are showed bythe direction 2 and 6 , also the principal diagonal direction are determined by the direction 1 and 5 and finallythe off diagonal direction code is masked by the 3 and 7 directions. Therefore, in every block, we acquired four values signifying the occurrences of these four ordersand these quantities were used as local contour direction featureset.For extracting these features, an unvarying block with $49(7 \times 7)$ size is considered in every image and we calculated four features (four directions) in each block so we acquired $49 \times 4=196$ features for every image.

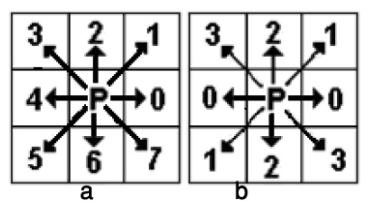

Figure 3. (a): chain code pattern for 8 direction (b): chain code pattern for 4 direction

\subsection{Modified Edge Maps Features}

In this stage, at the beginning, an $\mathrm{N} \mathrm{x} \mathrm{M}$ image is converted into the thinning form and then reshaped into a $49 \times 49$ matrix. For extracting the four distinct edge maps (horizontal, vertical and two diagonals $\left(45^{\circ}\right.$ and $\left.-45^{\circ}\right)$ ) the Sobel operators was used. After that these four distinct edge maps are distributed into 49 sub-images of $7 \times 7$ pixels. Then the featureset are gottencomputing the proportion of black pixels in each sub-image (a featureset with 49 dimension for each image). Finally these features are joint to form a single feature vector holding 196 (49 x 4) features.

\subsection{Transition features}

The third feature set that investigated in this paperis based on the extracting the transition value from background to foreground pixels in both vertical and horizontal directions. Our extracting The transition feature set is mostly like a transition feature set that hass been proposed by Kumar et al [19]. forextracting transition information, image is skimmed from top to bottom and left to right. Following actions shows the way of these feature extraction. 
Action1: Distribute the contoured image of a handwritten image into 49 part with size of $7 \times 7$.

Action2: Computethe number of transitions for each part and extract the 49 features for each character image.

\section{Classification by Ensemble TechniQue}

Ensemble technique has wonderful application in different techniques and widely used in pattern classification and machine learning. the diversekinds of ensemble techniques has been proposed before and among them theclassification fusion method is the most important type of the ensemble classification. In this technique, numerous classifiers are trained on a same feature space and then,the results of these classifiers are mixed to get a more precise classification [20]. In the current paper, we have used K-Nearest Neighbour (KNN), Linear (L) and Support Vector Machine (SVM) classifiers for ensemble technique. The features gained from Directional Chain Code (DCD) are applied to SVM, the Modified Edge Maps (MEM) feature Set is applied to linear classifiers and Transition features are applied to KNN separately. The prediction of these classifiers is combined using majority-voting procedure to appropriately classify the sample.

\subsection{Classification with use of the K-Nearest Neighbour}

If you faced with the classification problem has pattern classes you can use an efficient technique called the K-nearest neighbor classifier that display a reasonably limited degree of variability. With calculating the distance between the input pattern and the training patterns it could recognize each input pattern with certain accuracy that has given.During the classification only $\mathrm{k}$ nearest prototypes could takes into the input pattern.the final decision is performed with use of majority of class voting.In the k-Nearest neighbour technique, the distance among train and test set is computed for determining the class of the test set.In the Equation (1) the applying method is detailed:

$d=\sqrt{\sum_{k=1}^{\mathrm{N}}\left(x_{k}-y_{k}\right)^{2}}$

In the above equation, $\mathrm{x}_{\mathrm{k}}$ is the collection stored feature value, $\mathrm{N}$ shows the entire number of features in feature set and $y_{k}$ is the nominee feature value.

\subsection{Linear Classifier (LC)}

Linear classifier (LC) is a kind of the statistical classifier thatuses a value of the linear mixture of the featuresfor generating alabel of class.The application of the linear classifier is mostly on the circumstances where the speed of classification is an important issue.LCfrequentlyeffortexcellently when the number of magnitudes in feature vector is huge. It can be signified as revealed in equation (2):

$y=f(w, x)=f\left(\sum_{j} w_{j} x_{j}\right)$

Which $w_{j}$ is weight vector, learned from a set of marked training examples and thex $x_{j}$ is the feature vector of testing model and $\mathrm{f}$ is a simple function that applies the value to the individual classes based on a confident threshold. 


\subsection{Support Vector Machines (SVM) Classifier}

Support vector machines (SVMs) are one of the most importont, and powerfull in pattern learning and also in pattern recognition, because it support high dimensional data and at the same time, providing good generalization properties. Additionally, in data mining and pattern recognition applications, SVMs have very usages. Considered that $X=\left\{\left(x_{i}, y_{i}\right)\right\}^{n} i=1$ are samples of $n$ training, where, $y_{i} \in\{-1,1\}$ is a class label of sample xi, and $x_{i} \in R^{m}$ is an m-dimensional sample in the input space. with the minimal classification errors, SVM finds the optimal separating hyper plane (OSH). Equation (3) shown the linear separation hyper plane.

$f(x)=W^{T} x+b$

Here $\mathrm{b}$ and $W$ are the bias, and weight vector. By solving the optimization problem (6), the optimal hyper plane can be obtain, where variable $C$ controls the effect of the slack variables, and $\zeta_{i}$ is slack variable for obtaining a soft margin. Decreasing the value of $\mathrm{C}$ cause to increasing of separation margin. In a SVM, maximizing the generalization ability of the SVM cause to optimal hyper plane obtained. Anyhow, the obtained classifier may not have high generalization ability in a nonlinear separable training data. The original input space should be mapped into a highdimensional in order to enhancing the linear severability purpose. Now, with using the nonlinear vector function $\varphi(x)=\left(\varphi_{1}(x), \ldots, \varphi_{l}(x)\right)^{\Psi}$, witch maps the m-dimensional input vector $\mathrm{x}$ into the 1-dimensional feature space, the OSH in the feature space is given by equation (4):

$\mathrm{f}(\mathrm{x})=\mathrm{W}^{\mathrm{T}} \varphi(\mathrm{x})+\mathrm{b}$

Equation (5) defined a decision function for a test data:

$\mathrm{D}(\mathrm{x})=\operatorname{Sign}\left(\mathrm{W}^{\mathrm{T}} \varphi(\mathrm{x})+\mathrm{b}\right)$

By solving the following quadratic optimization problem, the optimal hyper plane can be found:

$\operatorname{Minimize} \frac{1}{2}\|\mathrm{~W}\|^{2}+\mathrm{C} \sum_{\mathrm{i}=1}^{\mathrm{n}} \zeta_{\mathrm{i}}$

Subject to $\mathrm{y}_{\mathrm{i}}\left(\mathrm{W}^{\mathrm{T}} \varphi(\mathrm{x})+\mathrm{b}\right) \geq 1-\zeta_{\mathrm{i}}$

$\zeta_{\mathrm{i}} \geq 0, \mathrm{i}=1, \ldots, \mathrm{n}$

As mentioned before, SVMs classifier isintroducedfor binary problemclassification, yet, our proposed method has more than two classes for classifications. For solving this problem, multiclass classification strategies that mentioned in [21] can be used. The greatestcommon ones are the one-against-one (OAO) and the one-against-all (OAA) approaches [22]. The one-againstone isusing the (n (n-1)) $/ 2$ equation for combinations of all class pairs. What achieved from the experimental results we find out that the one-against-all is more fitting for our proposed method. We used OAA for our character classification.

Our proposed classifier fusion method is sum up in the below algorithm. In addition Fig. 4 shows the recognition way for entrance test image by applying fusion of classifier. 


\section{Algorithm Begin:}

Input: a set of test samples and training samples.

Output: the class label for determining the test sample belongs.

Method: Step1: Extract the directional chain code (DCD), Modified Edge Maps (MEM) and transit (T) features for the training sample and test sample using previously discussed approaches respectively.

Step 2: Apply the DCD, MEM and T features obtained for the training samples to train the SVM, $\mathrm{KNN}$ and L classifiers respectively and separately.

Step 3: Apply the DCD, MEM and T features obtained for the test samples to each of the classifier.

Let the prediction of the classifiers be $\mathrm{p} 1, \mathrm{p} 2$ and $\mathrm{p} 3$

Step 4: Predict the class of the test sample as

Class $=$ Majority of the $\{\mathrm{p} 1, \mathrm{p} 2, \mathrm{p} 3\}$

End

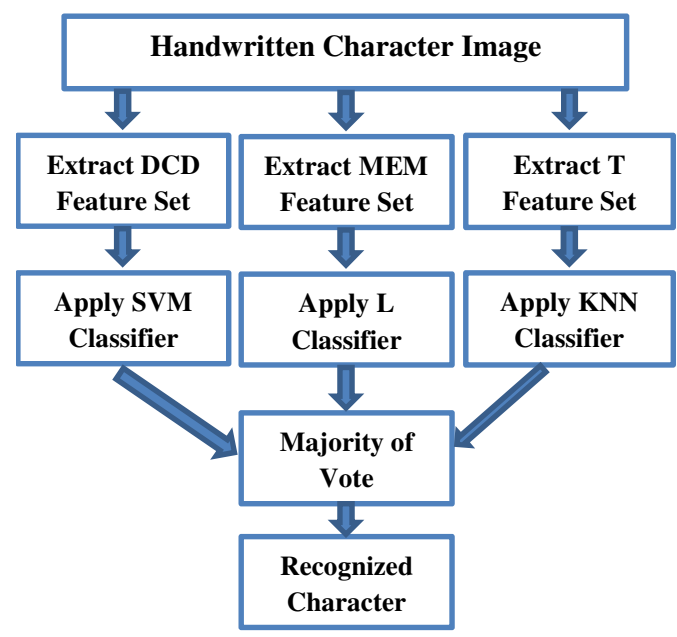

Figure 4. Architecture of the classifier fusion method to recognizehandwritten numerals

\section{Practical Results of the Proposed Method}

For analysing of the proposed method, a set of the 15,000 samples for training stage and a set of the 5,000 for test stage are considered as indicated in [23]. These samples contain Iranian Postal and National Codes and were extracted with use of the 200 dpi resolution scanner from different registration forms of the Iranian university entrance exam [24]. As we mentioned in section 2 the writing styles of different persons, samples sizes were extremely different, we standardized them to the constant size. By considering the 15,000 samples for the training stage, we evaluated our method on other 5,000 samples and we achieved $99.90 \%$ precision. What achieved from the experimental result, we obtained an exactness of $100 \%$ when the 20,000 data were utilized as 
training stage and the similar dataset was used for testing stage. For further analysing we separated our data set into 4 subclass and testing is estimated on each subclass using reminder of the 3 subsets for training stage. The mean of the recognition rates for all the four test subclass is achieved about $99.97 \%$. Table 1 shows the performance comparison of the proposed method with the state of the art methods.

Table 1. Recognition rates of the diverseapproachesfor recognizing the Persian handwritten numerals

\begin{tabular}{|l|c|c|c|c|}
\hline \multirow{2}{*}{\multicolumn{1}{|c|}{ Method }} & \multicolumn{2}{c|}{ Database size } & \multicolumn{2}{c|}{ Accuracy of the method (\%) } \\
\cline { 2 - 5 } & Train & Test & Train & Test \\
\hline Azad et al. [4] & 15000 & 5000 & - & 99.82 \\
\hline Shahreza et al.[5] & 2600 & 1300 & - & 97.80 \\
\hline Harifi et al. [6] & 230 & 500 & - & 97.60 \\
\hline Hosseini et al. [7] & 480 & 480 & - & 92.00 \\
\hline Mozaffari et al. [8] & 2240 & 1600 & 98 & 91.37 \\
\hline Rahmati et al.[9] & 4979 & 3939 & - & 99.57 \\
\hline Dehghan et al. [10] & 6000 & 4000 & - & 97.01 \\
\hline Faes et al. [11] & 6000 & 4000 & 100 & 97.65 \\
\hline Faes et al. [12] & 2240 & 1600 & 100 & 94.44 \\
\hline Mowlaei et al. [13] & 2240 & 1600 & 100 & 92.44 \\
\hline Mowlaei et al. [14] & 2240 & 1600 & 99.29 & 91.88 \\
\hline Sadri et al. [15] & 7390 & 3035 & - & 94.14 \\
\hline Parvin, et al [16] & 40000 & 2000 & - & 97.12 \\
\hline Parvin, et al [17] & 60000 & 10000 & - & 98.89 \\
\hline Our proposed method & 15000 & 50000 & 100 & 99.90 \\
\hline $\begin{array}{l}\text { Our proposed method } \\
\text { With 4 subset }\end{array}$ & 15000 & 50000 & 100 & 99.97 \\
\hline
\end{tabular}

For evaluation of the proposed method we used a dataset with 20,000 samples and weachieved $99.90 \%$ and $99.97 \%$ precisions using mentioned method. In our achievement with high accuracy (about $99.90 \%$ ), we detected confusion numerals in the recognition stageamong some digits. The main confusions were between 2, 4 and 3. This occurredsince 2, 3 and 4 resemble each other. In Fig. 5 the success and confusion rate for each character are depicted. 


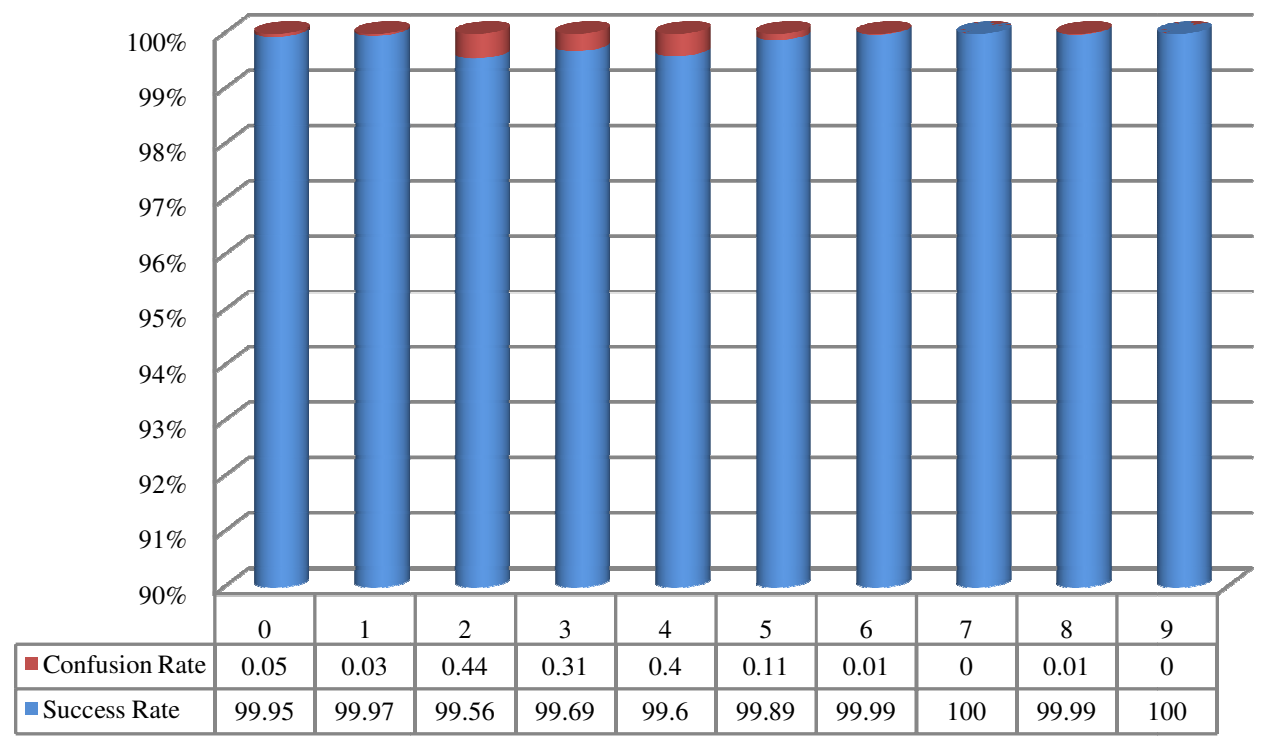

Figure 5. Success and confusion rate of the proposed approachfor each Persian numeral

\section{Conclusions}

In this paper, for robust Persian handwritten numerals recognition we have investigated three feature extraction approachessuch as directional chain code, modified edge maps and transit. For obtaining these feature set we transformed each image to the contour shape, then with use of block based method we achieved these three features sets. In the beginning, SVM, Linear and K nearest neighbour classifiers are used for the classification. Further, the recognition accuracy was enhancedwith use of classifier synthesis method. What achieved from the experimental results, it could be proofed that oursproposed techniquehas good performances on Persian handwritten numeral recognition. In addition, in the result part we detailedthe reason of the misclassification.Most of the misclassification samples on our method were from classes of 2, 3 and 4, which have similar structure.

\section{ACKNOWLEDGEMENTS}

This research is supported by the Shahid Rajaee Teacher Training University, Tehran, Iran (No.22970060-9).

\section{REFERENCES}

[1] A. Yuan, G. Bai, L. Jiao, Y. Liu, "Offline handwritten English character recognition based on convolutional neural network ", 10th IEEE International Workshop on Document Analysis Systems, 2012, pp. 125-129.

[2] S. N. Srihari and G. Ball, "An Assessment of Arabic Handwriting Recognition Technology", Springer book of Guide to OCR for Arabic Scripts, 2012, pp. 3-34.

[3] F. Yin, M. Zhou, Q. Wang and C. Liu, " Style Consistent Perturbation for Handwritten Chinese Character Recognition “, 12th IEEE International Conference onDocument Analysis and Recognition, 2013, pp. 1051-1055. 
[4] R. Azad, F. Davami and H. Shayegh, "Recognition of Handwritten Persian/Arabic Numerals Based on Robust Feature Set and K-NN Classifier", International Journal of Computer \& Information Technologies, Vol. 1, issue 3, 2013, pp. 220-230.

[5] M. H. Shirali-Shahreza, K. Faez and A. Khotanzad, "Recognition of Hand-written Persian/Arabic Numerals by Shadow Coding and an Edited Probabilistic Neural Network“, IEEE International Conference on Image Processing, Vol. 3, 1995, pp. 436-439.

[6] A. Harifi and A. Aghagolzadeh, "A New Pattern for Handwritten Persian/Arabic Digit Recognition", Journal of Information Technology Vol. 3, 2004, pp. 249-252.

[7] H. Mir Mohammad Hosseini and A. Bouzerdoum, "A Combined Method for Persian and Arabic Handwritten Digit Recognition", Australian New Zealand Conference on Intelligent Information System, 1996, pp. $80-83$.

[8] S. Mozaffari, K. Faez\& H. RashidyKanan, "Recognition of Isolated Handwritten Farsi/Arabic Alphanumeric Using Fractal Codes”, Image Analysis and Interpretation, 6th Southwest Symposium, 2004, pp. 104-108.

[9] H. Soltanzadeh and M. Rahmati, "Recognition of Persian handwritten digits using image profiles of multiple orientations", Pattern Recognition Letters 25, 2004, pp. 1569-1576.

[10] M. Dehghan and K. Faez, "Farsi Handwritten Character Recognition With Moment Invariants", Proceedings of 13th International Conference on Digital Signal Processing, Volume 2, 1997, pp. 507510.

[11] M. Ziaratban, K. Faez and F. Faradji, "Language-Based Feature Extraction Using Template-Matching in Farsi/Arabic Handwritten Numeral Recognition", Proceedings of 9th International Conference on Document Analysis and Recognition, Vol.1, 2007, pp. 297-301.

[12] S. Mozaffari, K. Faez and M. Ziaratban, "Structural Decomposition and Statistical Description of Farsi/Arabic Handwritten Numeric Characters", Proceedings of the 8th Intl. Conference on Document Analysis and Recognition, Vol. 1, 2005, pp. 237- 241.

[13] A. Mowlaei and K. Faez, "Recognition Of Isolated Handwritten Persiawarabic Characters And Numerals Using Support Vector Machines", Proceedings of XIII Workshop on Neural Networks for Signal Processing, 2003, pp. 547-554.

[14] A. Mowlaei, K. Faez\& A. Haghighat, "Feature Extraction with Wavelet Transform for Recognition of Isolated Handwritten Farsi/Arabic Characters and Numerals", Digital Signal Processing Vol. 2, 2002, pp. 923- 926.

[15] J. Sadri, C. Y. Suen and T. D. Bui, "Application of Support Vector Machines for Recognition of Handwritten Arabic/Persian Digits", Proceedings of the 2nd Conference on Machine Vision and Image Processing \& Applications, Vol. 1, 2003, pp. 300-307.

[16] H. Parvin, H. Alizadeh, M. Moshki, B. Bidgoli and N. Mozayani, ” Divide \& Conquer Classification and Optimization by Genetic Algorithm", Third 2008 International Conference on Convergence and Hybrid Information Technology, 2008, pp. $858-863$.

[17] H. Parvin, H. Alizadeh, B. Bidgoli and M. Analoui, "A Scalable Method for Improving the Performance of Classifiers in Multiclass Applications by Pairwise Classifiers and GA", Fourth International Conference on Networked Computing and Advanced Information Management , 2008, pp. $137-142$.

[18] R. Azad, B. Azad, "Real-Time Hand Gesture Recognition based on Modified Contour Chain Code Feature Set", IJIGSP, vol.6, no.8, pp.25-31, 2014.DOI: 10.5815/ijigsp.2014.08.04.

[19] M. Kumar, M. K. Jindal and R. K. Sharma, "k -Nearest Neighbor Based Offline Handwritten Gurmukhi Character Recognition," IEEE International Conference on Image Information Processing, pp.1-4, 2013.

[20] D. Parikh and R. Polikar, "An Ensemble-Based Incremental Learning Approach to Data Fusion”, IEEE Transaction on System, Man and Cybernetics, vol.37, no.2. April,2007 pp.437-450.

[21] F. Melgani and L. Bruzzone, "Classification of hyperspectral remote sensing images with support vector machine," IEEE Trans. Geosci. Remote Sens., vol. 42, no. 8, 2004, pp. 1778-1790.

[22] R. Azad, B. Azad and I. T. Kazeroni , "Optimized Method for Real-Time Face Recognition System Based on PCA and Multiclass Support Vector Machine”, Advances in Computer Science: an International Journal, Vol. 2, Issue 5, No.6 , November 2013, pp. 126-132. 
[23] R. Azad, F. Davami and H. Shayegh, "Recognition of Handwritten Persian/Arabic Numerals Based on Robust Feature Set and K-NN Classifier", International Conference on computer, Information Technology and Digital Media, 2013, pp. 161-165.

[24]H. Khosravi and E. Kabir, "Introducing a very large dataset of handwritten Farsi digits and a study on the variety of handwriting styles", Pattern Recognition Letters, 28(10), 2007, pp. 1133-1141.

\section{Authors}

Reza Azadobtained his B.Sc. degree with honor in computer software engineering from SRTTU in 2014. He is IEEE \& IEEE conference reviewer Member. Awarded as best student in 2013 and 2014 by the SRTTU and awarded as best researcher in 2013 by the SRTTU. He achieved fourth place in Iranian university entering exam. In addition he's a member of Iranian elites. He has a lot of scientific papers in international journal and

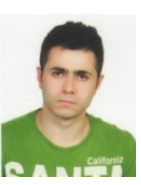
conferences, such as IEEE, Springer and etc. his interested research are artificial intelligence and computer vision.

Babak Azadis a researcher from Islamic Azad University. He achieved a lot of awards and publication on scientific papers in international journals and conferences, during his B.Sc. education. His most interest topics are machine learning and network.

Iraj Mogharreb is a B.Sc. student in Sabalan Higher educatin institute, Ardebil, Iran, in computer software engineering and he is top student in university. His research interests include image processing, machine learning and information security.

ShahramJamaliis currently an Associate Professor in Mohaghegh Ardabili University, Ardebil, Iran. He achieved his Ph.D degree in Architecture of Computer Systems in 2008 from Iran University of Science \& Technology, Tehran, Iran. He has more than 100 scientific papers in international journals and conferences, such as IEEE, Elsevier, Springer and etc. His research topics are Network security and soft computing.

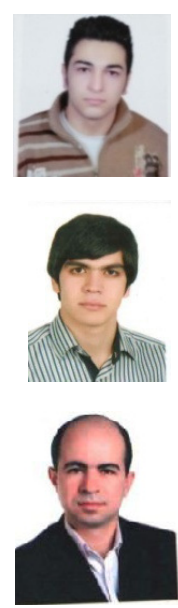

